Article

\title{
Online Identification with Reliability Criterion and State of Charge Estimation Based on a Fuzzy Adaptive Extended Kalman Filter for Lithium-Ion Batteries
}

\author{
Zhongwei Deng, Lin Yang *, Yishan Cai and Hao Deng \\ School of Mechanical Engineering, Shanghai Jiao Tong University, Shanghai 200240, China; \\ dengzhongw@126.com (Z.D.); cyshan2006@126.com (Y.C.); denghao5854@163.com (H.D.) \\ * Correspondence: yanglin@sjtu.edu.cn; Tel.: +86-139-1682-7881
}

Academic Editor: K. T. Chau

Received: 4 May 2016; Accepted: 13 June 2016; Published: 21 June 2016

\begin{abstract}
In the field of state of charge (SOC) estimation, the Kalman filter has been widely used for many years, although its performance strongly depends on the accuracy of the battery model as well as the noise covariance. The Kalman gain determines the confidence coefficient of the battery model by adjusting the weight of open circuit voltage (OCV) correction, and has a strong correlation with the measurement noise covariance $(R)$. In this paper, the online identification method is applied to acquire the real model parameters under different operation conditions. A criterion based on the OCV error is proposed to evaluate the reliability of online parameters. Besides, the equivalent circuit model produces an intrinsic model error which is dependent on the load current, and the property that a high battery current or a large current change induces a large model error can be observed. Based on the above prior knowledge, a fuzzy model is established to compensate the model error through updating $R$. Combining the positive strategy (i.e., online identification) and negative strategy (i.e., fuzzy model), a more reliable and robust SOC estimation algorithm is proposed. The experiment results verify the proposed reliability criterion and SOC estimation method under various conditions for $\mathrm{LiFePO}_{4}$ batteries.
\end{abstract}

Keywords: battery management system; intrinsic model error; parameter reliability criterion; fuzzy adaptive extended Kalman filter; state of charge

\section{Introduction}

To slow down the fossil fuel depletion and promote the hybridization and electrification of vehicle propulsion systems, electric vehicles (EVs) and hybrid electric vehicles (HEVs) are occupying more and more market share. Both of them need a battery management system (BMS) to guarantee safe and reliable battery operations. The key technology of a BMS is to monitor online the battery states, mainly including state of charge (SOC), state of health $(\mathrm{SOH})$, remaining useful life (RUL) and state of power (SOP). As a fundamental parameter of BMS, SOC determines the remaining energy in the battery pack and informs the driver how far the vehicle can still run at the current state. Since SOC cannot be measured directly during normal operation, a method to obtain accurate, real-time and robust SOC estimation is necessary and thus a hot research topic [1,2]. SOH describes the physical health condition of a battery compared to a fresh battery, and is usually defined as the ratio of the available capacity of a battery over its rated value. When its value is reduced to below $80 \%$, the battery is considered to be an unreliable power source and should be replaced [3]. Also indicating the battery degradation, RUL is defined as the period from the current time until the system or component fails. Estimation of the RUL contributes to the maintenance activities and prevents any accidents due to battery failure [4]. SOP is the parameter used to describe the maximum charging and discharging 
capabilities of the battery, and is very useful to formulate an optimal energy management strategy for vehicles. In this paper, we focus on SOC estimation.

The Ampere-Hour integral is the most widely used method to calculate SOC in real application, but uncertain initial SOC and the accumulated measurement errors are the accompanying weaknesses of this method [1,5]. The open circuit voltage (OCV) method makes use of the OCV-SOC relationship to find the SOC [6]. This method is accurate and reliable, but the battery needs a long time to relax so the method is thus unsuitable for real applications. Xing et al. [7] took the effect of temperature on the OCV-SOC curves into account and developed a temperature-based model to estimate SOC, and verified its higher accuracy at various ambient temperatures. Besides, the artificial neural network (ANN) [8], and support vector machine (SVM) [9] methods have been applied to construct black-box models for SOC estimation. The similar disadvantages of the above methods are the requirement for extensive experimental data to train the models. The most popular algorithm for SOC estimation is the extended Kalman filter (EKF) method, which combines the Ampere-Hour integral with open circuit voltage correction [10]. This method realizes the closed-loop estimation of SOC, and a variety of the analogous improved algorithms have been proposed, such as the adaptive extended Kalman filter (AEKF) [11], unscented Kalman filtering (UKF) [12,13] and adaptive unscented Kalman filtering (AUKF) [14]. The Kalman filter (KF) assumes the process and measurement noise are Gaussian, and the stable estimation can also be ensured, even when the noises are not exactly Gaussian. However, non-Gaussian noises have a negative influence on the convergence behavior and accuracy of the filter. To avoid this, Schwunk et al. [15] employed the particle filter (PF) to estimate the SOC and SOH of batteries and good accuracy was observed. It is noted that the SOC estimation accuracy of EKF strongly depends on the accuracy of the battery model as well as the noise covariance matrixes. According to the principle of the KF, the Kalman gain determines the confidence coefficient of the battery model by adjusting the weight of the open circuit voltage correction and has a strong correlation with the measurement noise covariance $(R)$. Lee et al. [16] demonstrated that the $\mathrm{R}$ can compensate the intrinsic model error taken by simplifying the battery model. They took the effect of battery current, current change and SOC value on the model error into consideration, and established a measurement noise model and data rejection method to continuously adjust $R$, and eventually improve the SOC estimation accuracy. As for obtaining noise covariance matrixes estimations, the AEKF based on covariance matching has been proposed and immediately offers superiority [17]. This method is simple in theory and with the advantage of small computational demand. Fuzzy logic has been used to establish the relationship between SOC and the electrochemical impedance spectroscopy (EIS) [18,19], and also estimate some battery parameters [20]. The fuzzy adaptive Kalman filter has been applied in inertial navigation systems and global positioning systems [21], and in this paper fuzzy models are mainly used to acquire accurate noise covariance, not to compensate the intrinsic model error.

In order to model the dynamic characteristics of battery, different forms of equivalent circuit models (ECMs) have been proposed $[16,22,23]$. Considering the trade-off between estimation accuracy and computational complexity, the Thevenin model has been widely used to realize SOC estimation. In terms of acquiring real battery parameters under different operation conditions and aging levels, positive strategies, namely online identification methods, are applied. The recursive least squares (RLS) algorithm [24,25], and EKF [26] have been employed to identify online the parameters of ECMs. The RLS algorithm does not require complex matrix operations such as inversion, but cannot be efficiently applied for strongly nonlinear battery models. The EKF can be used to estimate the parameters of nonlinear battery models, but needs more computing power, and it is sensitive to the initial parameters. Xiong et al. [24] derived a discrete time expression of the Thevenin model and employed the RLS method to identify online the model parameters. It is noted that the authors took the OCV as a known parameter, which is obtained by looking up the OCV-SOC curves, but the existing SOC deviations might lead to the identification of unreliable parameters. In our previous study [25], the OCV was regarded as a parameter needing identification, and the recursive extended least squares 
(RELS) algorithm was implemented to consider the existing colored noises and finally improve the accuracy of the model parameters.

In this work, we propose a fuzzy adaptive extended Kalman filter (FAEKF) to estimate SOC and the model parameters are identified online with a reliability criterion. The main contributions of this paper can be summarized as follows:

(1) The autoregressive moving average model with external input (ARMAX) of the battery is derived, which takes the OCV as a parameter needing identification. The zero-order hold discretization is implemented rather than the difference discretization to further improve the accuracy, and the RELS algorithm is used to realize the online identification.

(2) The OCV error between the offline and online value serves as an assessment criterion to judge the reliability of the online parameters. Making use of the property that the OCV-SOC curves remain almost invariant at different aging levels $[27,28]$, this method can apply to the whole life-cycle of the battery.

(3) A fuzzy model is established to update $R$ in real time to compensate the intrinsic model error, and the fuzzy rules are derived by using the verified prior knowledge.

(4) The positive strategy based on an online identification strategy and the negative strategy using a fuzzy model to update $R$ are combined to improve the SOC estimation accuracy.

The remainder of this paper are arranged as follows: Section 2 introduces the battery model structure and the intrinsic model error; Section 3 describes the online identification method and the proposed reliability criterion for online parameters; the theory of FAEKF for SOC estimation is illustrated in Section 4; the experimental results and discussion are presented in Section 5; Section 6 concludes this paper.

\section{Equivalent Circuit Model}

In order to employ model-based algorithms to estimate SOC, an appropriate model of the battery is needed. A variety of battery models have been developed to capture the characteristics of lithium-ion batteries for different purposes; among them the ECMs with one or two RC ladders are widely used in automobile studies [23]. Considering the computational complexity and accuracy of the model, the Thevenin model as shown in Figure 1 is the most widely used. The model error is the voltage error between the measured terminal voltage and the model output value operating under the same conditions. Since the Thevenin model is a simplified battery model, an intrinsic model error exists. The influence of the battery current on the model error is analyzed in Section 2.2.

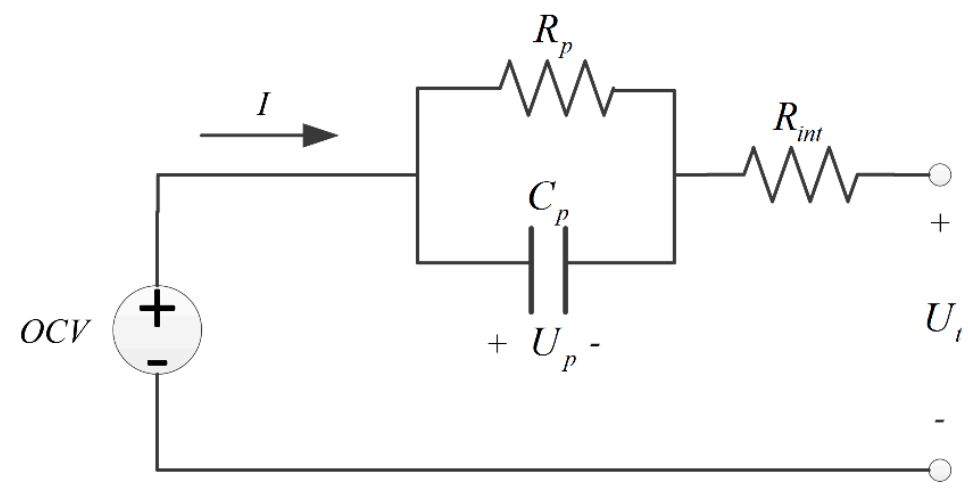

Figure 1. The structure of the Thevenin model.

\subsection{Thevenin Model}

As illustrated in Figure 1, $R_{\text {int }}$ is the series resistance, which models the charge transfer, double layer and internal resistance of the battery due to their very fast dynamics. The first-order RC 
network is used to model the diffusion phenomena of the battery because of its relatively slower dynamics [16]. The OCV stands for the equilibrium potential of the battery, and it's a function of SOC and battery temperature. Load current $I$ is the input variable (suppose positive for discharge, negative for charge), and terminal voltage $U_{t}$ is the output variable. The battery model can be expressed by the following equations:

$$
\begin{gathered}
\dot{U}_{p}=-\frac{U_{p}}{C_{p} R_{p}}+\frac{I}{C_{p}} \\
U_{t}=U_{o c}-U_{p}-R_{i n t} I
\end{gathered}
$$

where $U_{p}$ is the terminal voltage of the RC network; $U_{o c}$ is the mean value of OCV at charge and discharge conditions.

\subsection{Model Error}

To illustrate the variation of model error along with the battery current, the offline pulse identification method is firstly conducted to acquire the parameters of the Thevenin model. The schematics of the offline pulse identification method are shown in Figure $2 \mathrm{a}, \mathrm{b}$ and an improved form of this method is presented in [29]. The OCV is determined from the steady-state voltage after the pulse discharge. The model parameters for a $\mathrm{LiFePO}_{4}$ battery acquired by offline identification at $20{ }^{\circ} \mathrm{C}$ operating conditions are listed in Table 1 . The OCV-SOC curves of the $\mathrm{LiFePO}_{4}$ battery at different ambient temperatures are shown in Figure 2c, and these curves both have a relatively flat region (i.e., OCV plateau), making the OCV correction unreliable in that area.
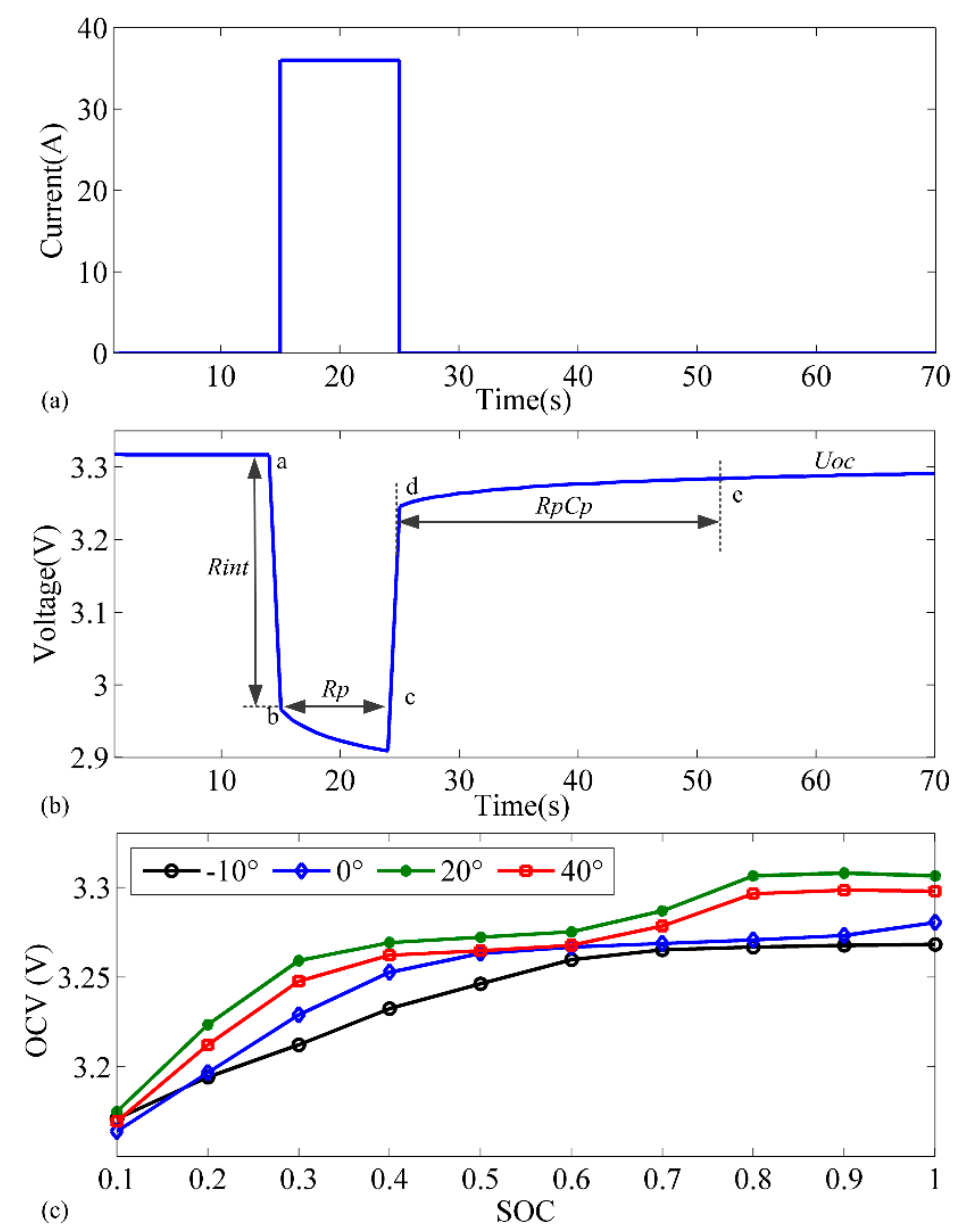

Figure 2. The offline pulse identification method for the Thevenin model. (a) pulse current; (b) voltage response; (c) OCV-SOC curves. 
Table 1. The parameters of the Thevenin model acquired by offline identification at $20{ }^{\circ} \mathrm{C}$.

\begin{tabular}{ccccccccccc}
\hline SOC & 0.1 & 0.2 & 0.3 & 0.4 & 0.5 & 0.6 & 0.7 & 0.8 & 0.9 & 1 \\
OCV (V) & 3.175 & 3.224 & 3.259 & 3.269 & 3.272 & 3.275 & 3.282 & 3.300 & 3.305 & 3.311 \\
$\operatorname{Rint}(\mathbf{m} \Omega)$ & 10.19 & 9.94 & 9.89 & 9.83 & 9.78 & 9.78 & 9.78 & 9.78 & 9.8 & 9.83 \\
$\operatorname{Rp}(\mathbf{m} \Omega)$ & 6.322 & 5.454 & 4.762 & 5.027 & 5.206 & 4.899 & 5.465 & 6.515 & 7.029 & 7.313 \\
$\mathbf{C p}(\mathbf{F})$ & 6096 & 5715 & 5858 & 6628 & 6708 & 7077 & 6824 & 6190 & 6354 & 6487 \\
\hline
\end{tabular}

Some experiments under the same conditions are conducted to analyze the influence of the battery current and current change on the model error. Considering the characteristics of the battery, the amplitude of the discharge current is twice that of the charge current. The model errors at different constant currents are shown in Figure 3a,b, and the mean model errors are listed in Table 2. The results confirm that a higher current leads to a larger model error. In order to compare the effect of different current changes on the model error, some special current profiles with a same average value are designed. The same average value of current is employed to eliminate the influence of current amplitude. The model errors caused by different current changes are presented in Figure $3 c, d$, and the mean model errors are listed in Table 2 . The results validate that a faster changing current leads to a larger model error, and are consistent with the results in [16]. The model error will decrease the SOC estimation accuracy of model-based algorithms, and even lead to the non-convergence of the estimator. A fuzzy model based on the prior knowledge is established to compensate the model error in Section 4.
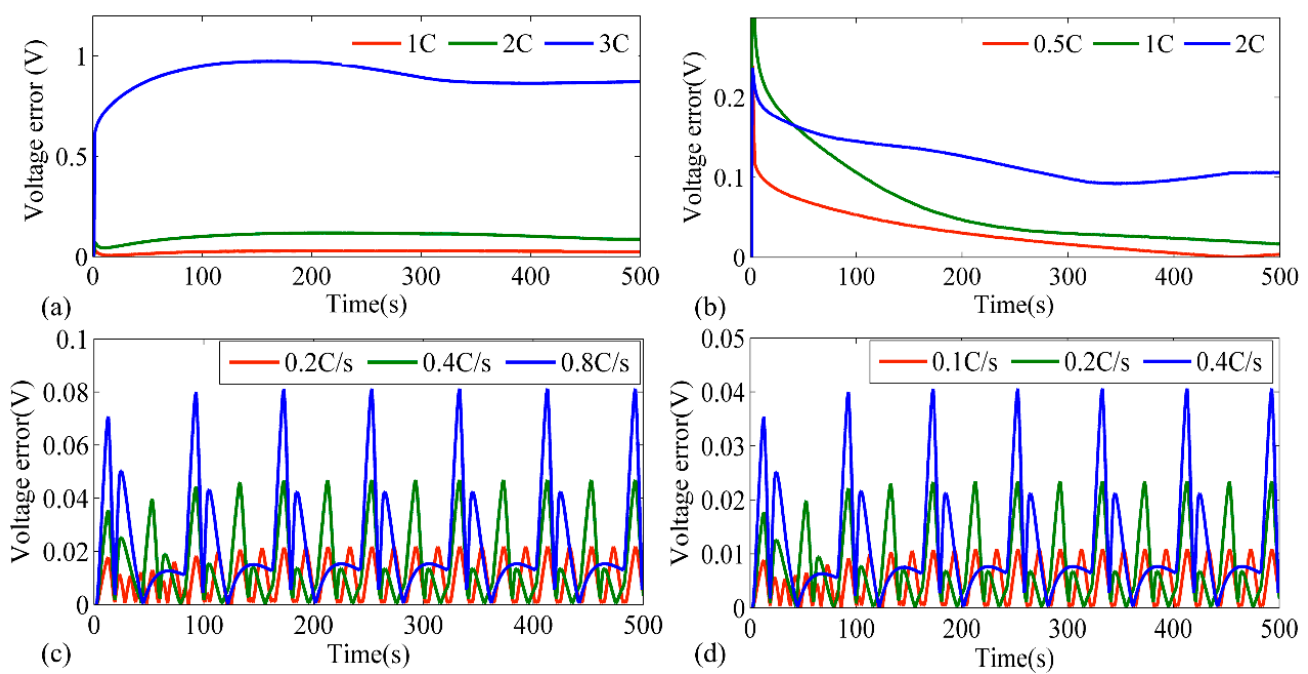

Figure 3. The model errors at different current profiles. (a) constant current discharge; (b) constant current charge; (c) changing current discharge; (d) changing current charge.

Table 2. The mean model errors caused by the different current profiles.

\begin{tabular}{ccccc}
\hline Condition & Constant Current & Mean Model Error & Current Change & Mean Model Error \\
\hline \multirow{3}{*}{ Discharge } & $1 \mathrm{C}$ & $0.0249 \mathrm{~V}$ & $0.2 \mathrm{C} / \mathrm{s}$ & $0.0096 \mathrm{~V}$ \\
& 2C & $0.1007 \mathrm{~V}$ & $0.4 \mathrm{C} / \mathrm{s}$ & $0.0160 \mathrm{~V}$ \\
\multirow{4}{*}{ Charge } & 3C & $0.8978 \mathrm{~V}$ & $0.8 \mathrm{C} / \mathrm{s}$ & $0.0231 \mathrm{~V}$ \\
& $0.5 \mathrm{C}$ & $0.0303 \mathrm{~V}$ & $0.1 \mathrm{C} / \mathrm{s}$ & $0.0048 \mathrm{~V}$ \\
& $1 \mathrm{C}$ & $0.0640 \mathrm{~V}$ & $0.2 \mathrm{C} / \mathrm{s}$ & $0.0080 \mathrm{~V}$ \\
& 2C & $0.1220 \mathrm{~V}$ & $0.4 \mathrm{C} / \mathrm{s}$ & $0.0114 \mathrm{~V}$ \\
\hline
\end{tabular}

\section{Proposed Online Identification Strategy for ECM}

As model parameters vary with the operation conditions and aging levels, an online identification method is necessary to acquire the real parameters during the whole life-cycle of the battery. 
In practical application, online parameters are not always believable due to the complicated driving cycle. The reliability of online identified parameters needs to be judged before using them in model-based algorithms.

\subsection{Online Identification Method}

Before applying the online identification algorithms, a single input single output (SISO) discrete system of the battery model needs to be derived. For pursue high accuracy battery parameters, a more accurate discretization method (i.e., zero-order hold discretization) is implemented rather than the difference discretization used in our previous study [25]. The discrete time expression of the Thevenin model can be obtained stepwise. By discretizing the state equation of the battery model in Equation (1), a linear discrete form can be acquired as:

$$
\begin{gathered}
U_{p, k+1}=\exp \left(-\frac{\Delta t}{\tau}\right) U_{p, k}+\left(1-\exp \left(-\frac{\Delta t}{\tau}\right)\right) R_{p} I_{k} \\
U_{t, k+1}=U_{o c}-U_{p, k+1}-R_{i n t} I_{k+1}
\end{gathered}
$$

where $\Delta t$ is fixed sampling period and time constant $\tau=R_{p} C_{p}$. Combining Equations (2) and (3), we can get the complete response of the Thevenin model as:

$$
U_{t, k+1}=U_{o c}-\left(\exp \left(-\frac{\Delta t}{\tau}\right) U_{p, k}+\left(1-\exp \left(-\frac{\Delta t}{\tau}\right)\right) R_{p} I_{k}\right)-R_{\text {int }} I_{k+1}
$$

Using the previous state of Equation (3), we can eliminate $U_{p, k}$ and get:

$$
U_{t, k+1}=\exp \left(-\frac{\Delta t}{\tau}\right) U_{t, k}+\left(1-\exp \left(-\frac{\Delta t}{\tau}\right)\right) U_{o c}-R_{i n t} I_{k+1}+\left(\exp \left(-\frac{\Delta t}{\tau}\right) R_{i n t}-\left(1-\exp \left(-\frac{\Delta t}{\tau}\right)\right) R_{p}\right) I_{k}
$$

The colored noise $e(k+1)$ can be expressed by a sequence of white noises $\xi(k+1)$, namely:

$$
\mathrm{e}(k+1)=\xi(k+1)+c_{1} \xi(k)+c_{2} \xi(k-1)+\cdots+c_{n_{c}} \xi\left(k+1-n_{c}\right)
$$

Combining Equations (5) and (6), we can get the ARMAX model of the battery as:

$$
\begin{aligned}
U_{t, k+1} & =\exp \left(-\frac{\Delta t}{\tau}\right) U_{t, k}+\left(1-\exp \left(-\frac{\Delta t}{\tau}\right)\right) U_{o c}-R_{\text {int }} I_{k+1}+\left(\exp \left(-\frac{\Delta t}{\tau}\right) R_{\text {int }}-\left(1-\exp \left(-\frac{\Delta t}{\tau}\right)\right) R_{p}\right) I_{k} \\
& +\xi(k+1)+c_{1} \xi(k)+c_{2} \xi(k-1)+\cdots+c_{n_{c}} \xi\left(k+1-n_{c}\right) \\
& =\boldsymbol{\Phi}_{k+1} \theta^{T}+\xi(k+1)
\end{aligned}
$$

where:

$$
\begin{aligned}
\boldsymbol{\phi}_{k+1} & =\left[U_{t, k}, 1, I_{k+1}, I_{k}, \xi(k), \xi(k-1), \cdots, \xi\left(k+1-n_{c}\right)\right] \\
\theta & =\left[\exp \left(-\frac{\Delta t}{\tau}\right),\left(1-\exp \left(-\frac{\Delta t}{\tau}\right)\right) U_{o c},-R_{\text {int }}, \exp \left(-\frac{\Delta t}{\tau}\right) R_{\text {int }}-\left(1-\exp \left(-\frac{\Delta t}{\tau}\right)\right) R_{\text {int }}, c_{1}, c_{2}, \cdots, c_{n_{c}}\right] \\
& =\left[a_{1}, a_{2}, a_{3}, a_{4}, c_{1}, c_{2}, \cdots, c_{n_{c}}\right]
\end{aligned}
$$

From Equation (8), the model parameters can be obtained as:

$$
U_{o c}=\frac{a_{2}}{1-a_{1}}, R_{i n t}=-a_{3}, R_{p}=\frac{a_{4}+a_{1} a_{3}}{a_{1}-1}, C_{p}=\frac{\Delta t\left(1-a_{1}\right)}{\left(a_{4}+a_{1} a_{3}\right) \log \left(a_{1}\right)}
$$

Then we can employ the RELS algorithm with forgetting factor to realize the online parameter identification. The forgetting factor gives higher weight to new data so that the algorithm can avoid the phenomenon of data saturation. Figure 4 shows the flow chart of the RELS algorithm. The initial value of the parameter estimation $\theta(0)$ is set as the offline value, and its error covariance matrix $\boldsymbol{P}(0)$ is set as unit matrix. Since $\xi(k+1)$ is unmeasurable, we use its estimation value $\hat{\xi}(k+1)$ to substitute it, which can be calculated as:

$$
\hat{\xi}(k+1)=U_{t, k+1}-\hat{\phi}_{k+1}^{T} \hat{\theta}_{k+1}
$$


The initial sequence $\xi(k), \xi(k-1), \cdots, \xi\left(k+1-n_{\mathcal{c}}\right)$ is set as zero. A new estimation value is added into the position of the first element and the last element is deleted at every step.

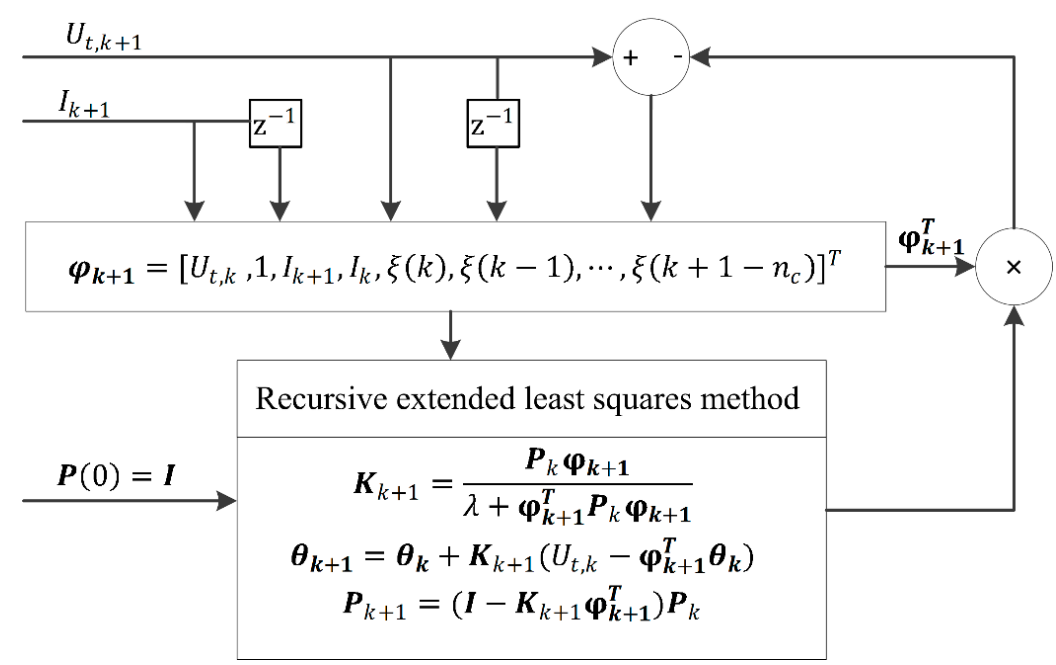

Figure 4. The flow chart of the RELS algorithm.

For validating the proposed online identification model, we need battery current and voltage profiles to apply the RELS algorithm. The urban dynamometer driving schedule (UDDS) cycle is a typical driving cycle which is widely used to evaluate the battery model and state estimation algorithms. The current profile of the UDDS cycle is extracted from an electric vehicle, and then loaded into the battery to generate the corresponding voltage response. The UDDS cycle current and voltage profiles are shown in Figure 5a.

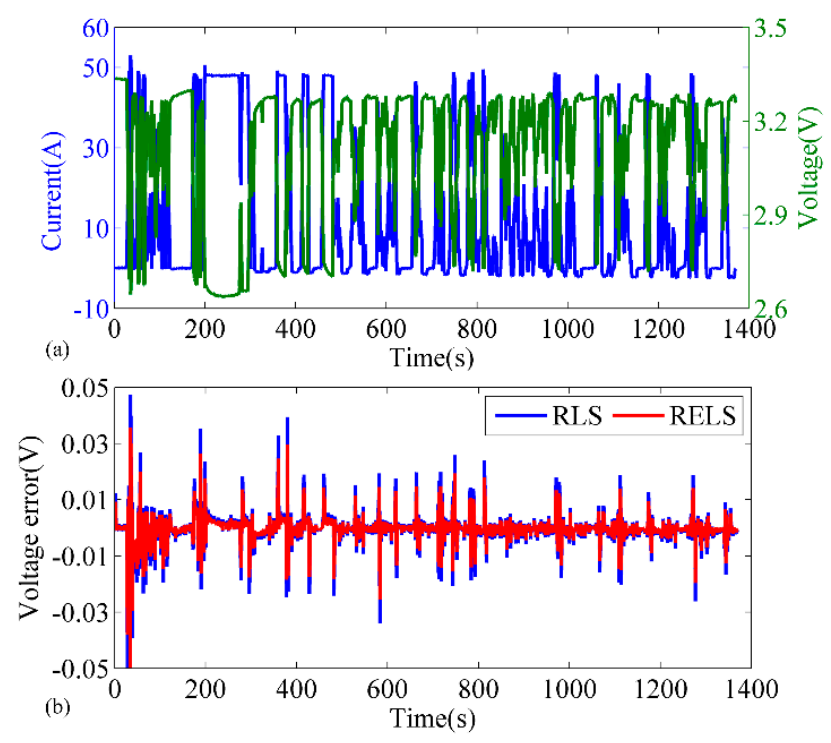

Figure 5. The online identification results of the RELS method. (a) UDDS profiles; (b) voltage errors.

To evaluate the proposed online identification method, the model errors caused by the RELS are compared with that by the RLS and are presented in Figure 5b. The maximum error (MaxE) of the RLS is $47.3 \mathrm{mV}$ and the root mean square error (RMSE) of the RLS is $6.8 \mathrm{mV}$. While the MaxE of the RELS is $35.5 \mathrm{mV}$ and the RMSE of the RELS is only $5.1 \mathrm{mV}$. These results verify that the RELS taking the existing colored noises into consideration can capture the battery dynamic behaviors more effectively than the RLS. 


\subsection{Parameters Reliability Criterion}

In practical application, the driving cycle is complicated by different operating conditions, so the reliability of online parameters needs to be judged before using in SOC estimation. As illustrated in Figure 5b, the model errors are very large at some points. Besides, large deviations exist during operation under specific operating conditions, such as constant current condition and pulse current condition, since the simple current profiles cannot inspire the complete dynamics of the battery system. As mentioned above, the parameter $U_{o c}$ has been identified by the RELS method. Generally, the identified $U_{o c}$ cannot be used to get SOC according to the OCV-SOC curves, because the small error of $U_{o c}$ can cause large SOC errors. Since $U_{o c}$ is more sensitive than other parameters and changes during a narrow range, so we can use it to evaluate the reliability of online parameters. If the $U_{o c}$ acquired by online and offline identification satisfy the Equation (11), we think the obtained online parameters are reliable and can be used in SOC estimation:

$$
\left|U_{o c \_o f f l i n e}-U_{o c \_o n l i n e}\right| \leqslant \Delta \mathrm{mV}
$$

where, the parameter $\Delta$ needs to be calibrated through experiments for different type of batteries. If the $\Delta$ is too small, the online parameters cannot update in a timely way according to the operating conditions; if the $\Delta$ is too large, the online parameters could not capture the battery characteristics. Since the OCV-SOC curves remain almost invariant at different aging levels $[27,28]$, this proposed reliability criterion can be suitable for the battery at different aging levels. The offline identification $U_{o c}$ can be obtained by using the previous estimated SOC to look it up in Table 1 at the corresponding temperature. This reliability criterion might be applied to different types of lithium-ion batteries by searching for an optimal $\Delta$.

In order to analyze the influence of operating conditions, the online parameters identification results for $\mathrm{LiFePO}_{4}$ batteries at constant current and pulse current condition are presented in Figure 6 . The legend of NO- $R$ stands for the results without using the reliability criterion, while the legend of $R$ represents the results using the reliability criterion with $\Delta=12$. The drastic changes and non-convergence of the online parameters verify the poor performance of online identification method at these specific conditions. While the reliability criterion can get rid of unreliable parameters and keep the acquired parameters convergent, thus improve the accuracy of the model parameters.
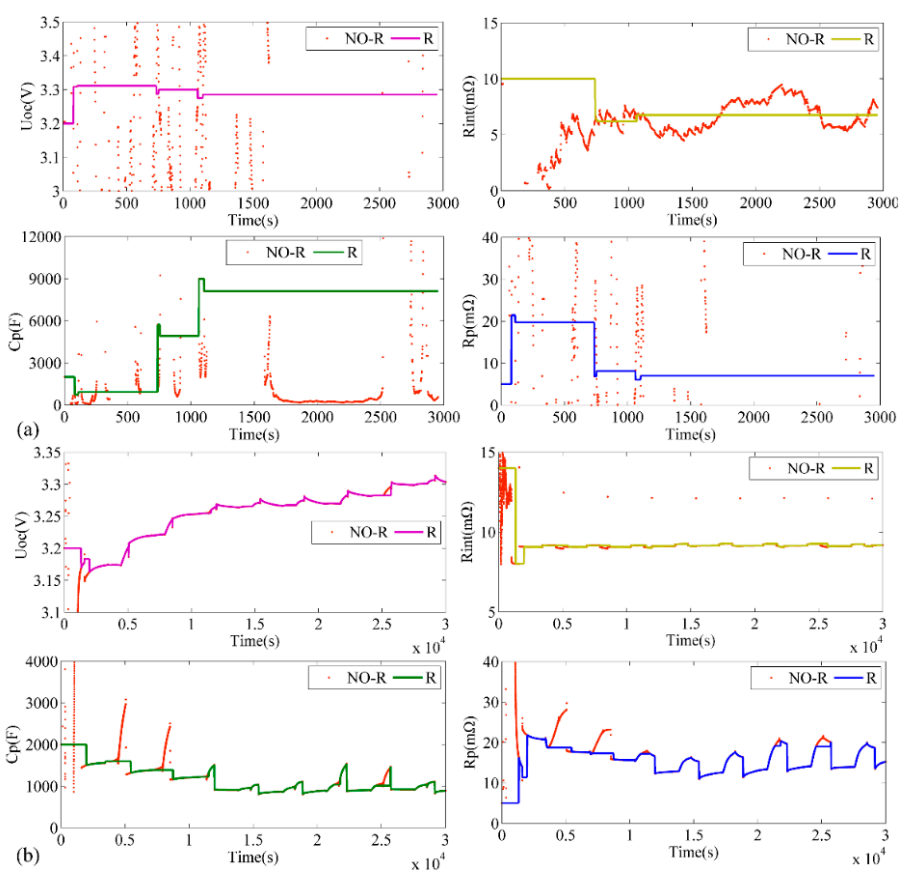

Figure 6. The online parameters identification results at constant current and pulse current conditions. (a) 1C constant current; (b) pulse current. 


\section{Fuzzy Adaptive Extended Kalman Filter for SOC Estimation}

As described above, the performance of the Kalman filter strongly depends on the accuracy of the battery model as well as the noise covariance. The online identification strategy aims to acquire the real model parameters under different operating conditions, while the fuzzy model discussed in this section intends to obtain more valuable noise covariance to compensate the intrinsic model error. It has been proved that the Artificial Neural Network (ANN) can approximate any nonlinear function in arbitrary precision; however, the network requires huge datasets for training, and is susceptible to local minimum points. Since the nonlinear prior knowledge has been acquired, the fuzzy logic can model the nonlinear relationship, and the derived fuzzy reasoning tables can be conveniently stored in the controller memory, so in this section, we propose the FAEKF algorithm, which modifies the AEKF by using fuzzy model to update $R$, while the process noise covariance $(Q)$ is still estimated by the innovation sequence. After simply introducing the principle of AEKF, the way of how to establish the proposed fuzzy model will be presented in this section.

\subsection{Adaptive Extended Kalman Filter}

Before employing the AEKF algorithm for the SOC estimation, the state transition and measurement update equations that relate the SOC to the voltage need to be derived. The SOC is defined as a ratio of the remaining capacity over the maximum available capacity, and a discrete time form of the SOC function is expressed as:

$$
S O C_{k+1}=S O C_{k}-\eta \frac{I_{k} \Delta t}{C_{N}}
$$

where $C_{N}$ is the maximum available capacity of battery, $\eta$ is the coulomb efficiency which is approaching 1 for lithium-ion battery. The model of lithium-ion battery belongs to the nonlinear system, so its state equations can be expressed as:

$$
\begin{gathered}
x_{k+1}=f\left(x_{k}, I_{k}\right)+w_{k} \\
z_{k}=g\left(x_{k}, I_{k}\right)+v_{k}
\end{gathered}
$$

where $w_{k}, v_{k}$ are process noise and measurement noise with zero-means respectively, $f(\cdot), g(\cdot)$ are the nonlinear functions. By combining Equations (1), (12), and (13) and executing linearization, we get the state transition and measurement update equations as:

$$
\begin{aligned}
& x_{k+1}=\left[\begin{array}{l}
S O C_{k+1} \\
U_{p, k+1}
\end{array}\right]=G x_{k}+H I_{k}+w_{k} \\
& z_{k}=U_{o c}(S O C)-U_{p, k}-R_{\mathrm{int}} I_{k}+v_{k}=C x_{k}-R_{\mathrm{int}} I_{k}+v_{k}
\end{aligned}
$$

where:

$$
G=\left[\begin{array}{cc}
1 & 0 \\
0 & \exp \left(-\frac{\Delta t}{\tau}\right)
\end{array}\right], H=\left[\begin{array}{c}
-\eta \frac{\Delta t}{C_{N}} \\
\left(1-\exp \left(-\frac{\Delta t}{\tau}\right)\right) R_{\mathrm{p}}
\end{array}\right], C=\left[\left.\frac{d U_{o c}(S O C)}{d S O C}\right|_{S O C_{k}}-1\right]
$$

As illustrated in Figure 2c, the OCV-SOC curves gradually vary with the ambient temperature, so a precomputed 2D-table with SOC and temperature as inputs is stored to look up the C matrix. The formulas for calculating the measurement noise covariance estimation $\hat{R}_{k}$ and the process noise covariance estimation $\hat{Q}_{k}$ are derive in [30] and can be concluded as follows:

$$
\mathrm{e}_{k}=z_{k}-g\left(\hat{x}_{k}^{-}, I_{k}\right), \hat{R}_{e_{k}}=\frac{1}{m} \sum_{i=k-m+1}^{k} \mathrm{e}_{i} \mathrm{e}_{i}^{T}, \hat{R}_{k}=\hat{R}_{e_{k}}-C_{k} P_{k}^{-} C_{k}^{T}, \hat{Q}_{k}=K_{k} \hat{R}_{e_{k}} K_{k}^{T}
$$


where $\mathrm{e}_{k}$ is the innovation sequence, and $m$ is the size of moving estimation window. $K_{k}, C_{k}$ are the Kalman gain and $\mathrm{C}$ matrix at kth sampling time, and $P_{k}^{-}$is the covariance estimation of the state error. Comparing with the EKF, the AEKF algorithm based on the covariance matching uses the real-time noise covariance estimation to replace the fixed values.

\subsection{How to Establish the Fuzzy Model}

As verified in Section 2.2, a high battery current or a large current change induces a large model error. Lee et al. [16] attributed the above fact to the simplified ECM, which cannot efficiently capture the fast dynamics of a battery including charge transfer, double layer and diffusion, and thus enough model errors are caused when the battery current is large or changing fast. They also demonstrated that this intrinsic model error can be compensated by reasonably modifying $R$ when employing the KF algorithm for SOC estimation. Besides, for the $\mathrm{LiFePO}_{4}$ battery studied in this paper, the OCV-SOC curves have a relatively flat middle region (i.e., OCV plateau), making the OCV correction unreliable in this area. According to above analysis, the input variables of the fuzzy model can be battery current, current change and SOC, and the output variable of the fuzzy model is $R$ obviously.

The fuzzy rules are established based on below strategies. When the model error is large (i.e., large or fast changing current), the $R$ correspondingly increases to enlarge the weight of the Ampere-Hour integral; when the model error is small (i.e., small or slow changing current), the $R$ correspondingly decreases to enlarge the weight of OCV correction. A For $\mathrm{LiFePO}_{4}$ battery with OCV plateau, in the SOC range of $40 \%-70 \%$, the $R$ is increased to enlarge the weight of the Ampere-Hour integral; while at the two ends of SOC range, the $R$ is decreased to enlarge the weight of the OCV correction. The actual inputs of the fuzzy model are the absolute value of battery current $(I)$, current change $(\Delta I)$ and SOC, and the output is $R$, while the $Q$ is still estimated by the innovation sequence. According to the maximum discharge current of the battery, the range of $I$ is $(04 \mathrm{C})$. Referring to the current map in the UDDS cycle, the range of $\Delta \mathrm{I}$ is $(03 \mathrm{C})$. The range of the $R$ is $(08)$, the upper bound is obtained by repeatedly simulating the algorithm with the object of the lowest SOC estimation error. For different capacity $\mathrm{LiFePO}_{4}$ batteries, the parameter that needs to be calibrated is the upper bound of $R$. The membership functions of inputs and output are presented in Figure $7 a$, and the surfaces of the fuzzy rules are shown in Figure $7 \mathrm{~b}$. The $I, \Delta I$ and SOC are divided into three ranks namely "Small", "Medium" and "Large" by using linguistic variables, while $R$ is divided into nine levels. The membership function of SOC is Gaussian combinational membership functions, which can effectively take the OCV plateaus of the OCV-SOC curves into consideration. The membership functions of other inputs and output are common triangular shaped functions.

The flow chart of the proposed FAEKF algorithm is illustrated in Figure 8. The real-time acquisition of current and voltage data are input into the RELS algorithm to online identify the model parameters; then the reliability of the online parameters is evaluated by using the proposed criterion, and the reliable parameters are input into the Thevenin model to update matrix $G, H$ and C; the battery current data and estimated SOC are input into the fuzzy model to output $R$ through the fuzzy logic reasoning, and the $Q$ is constructed by the innovation sequence; the time update equations and measurement update equations are implemented to compute new state variables. 

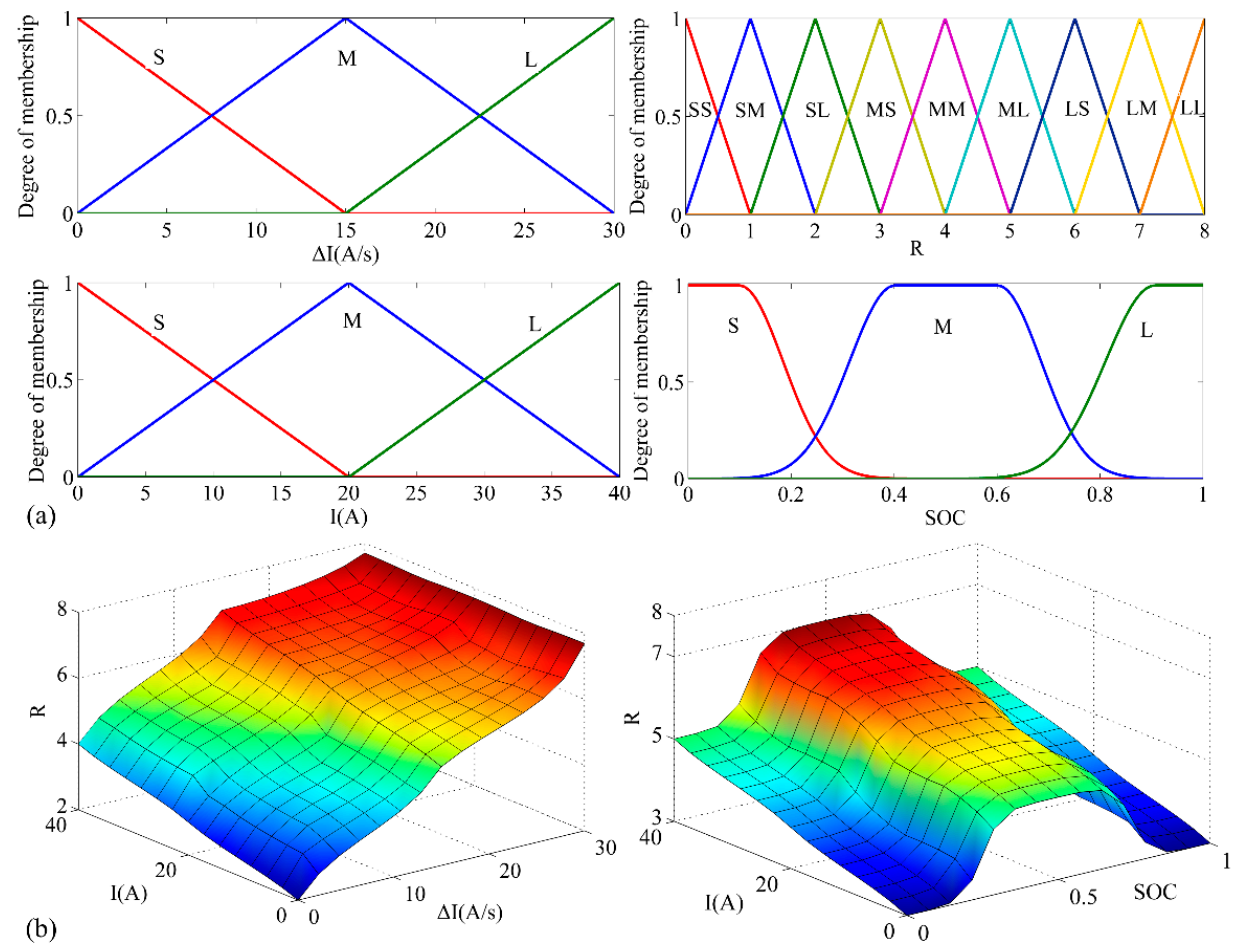

Figure 7. The fuzzy model for updating $R$. (a) fuzzy membership functions; (b) the surface of the fuzzy rules.

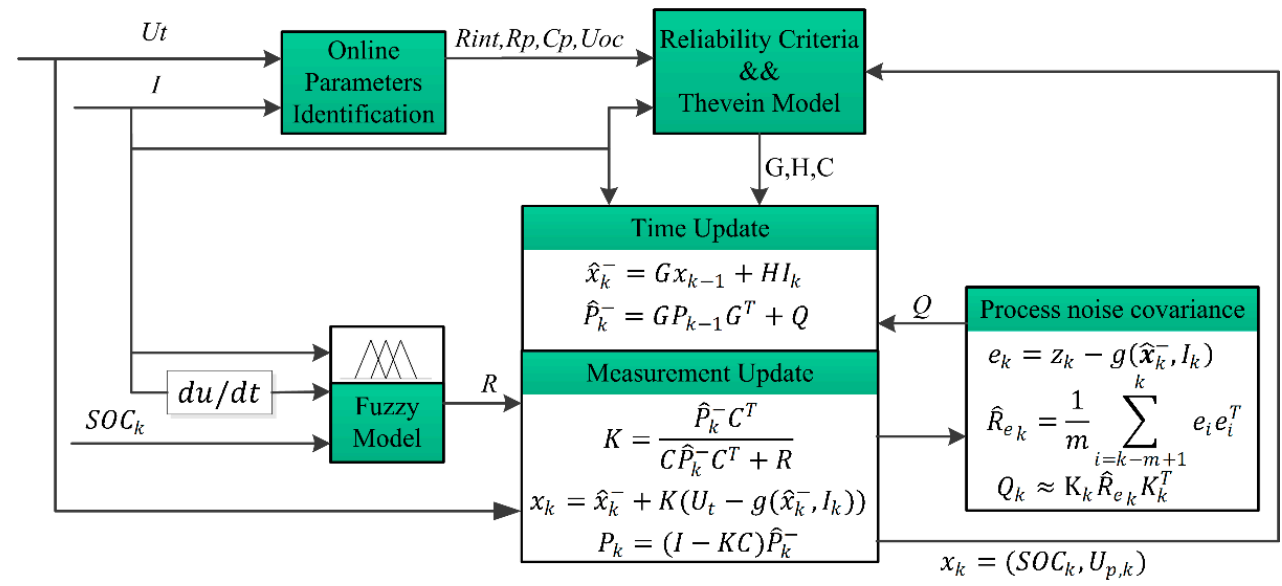

Figure 8. The flow chart of the proposed FAEKF algorithm.

\section{Experiment Results and Discussion}

The test bench consists of a UBT 300-060 multifunctional battery tester (Digatron, Qingdao, Shandong, China), a thermal chamber, a personal computer and some $10 \mathrm{Ah} \mathrm{LiFePO}_{4}$ batteries. Some specifications of the Digatron UBT 300-060 are presented at Table 3, while the characteristic parameters of the $\mathrm{LiFePO}_{4}$ batteries are shown in Table 4. The UBT300-060 includes voltage, current and temperature sensors, and a computer interface. It can charge/discharge with a maximum current of $300 \mathrm{~A}$, and can be set by the program to work for a variety of patterns, such as constant voltage mode, constant current mode and driving cycle mode. The accompanying Digatron BTS 600 software can realize procedural control of the charge/discharge process, and automatically record the battery parameters, such as charge and discharge duration, current, voltage, temperature, power and ampere hours. The thermal chamber provides constant ambient temperature for the experiments. 
Table 3. Specifications of the Digatron UBT 300-060 universal battery tester.

\begin{tabular}{lc}
\hline \multicolumn{1}{c}{ Feature } & Value \\
\hline Maximum discharge current & $300 \mathrm{~A}$ \\
Maximum charge current & $300 \mathrm{~A}$ \\
Voltage range & $0-60 \mathrm{~V}$ \\
Current sampling error & $\pm 50 \mathrm{~mA}$ \\
Voltage sampling error & $\pm 5 \mathrm{mV}$ \\
Temperature sampling error & $\pm 0.1 \mathrm{~K}$ \\
\hline
\end{tabular}

Table 4. Characteristic parameters of the $\mathrm{LiFePO}_{4}$ batteries.

\begin{tabular}{lc}
\hline \multicolumn{1}{c}{ Parameter } & Value \\
\hline Rated capacity & $10 \mathrm{Ah}$ \\
Nominal voltage & $3.2 \mathrm{~V}$ \\
Maximum discharge current (continuous) & $36 \mathrm{~A}$ \\
Maximum charge current (continuous) & $24 \mathrm{~A}$ \\
Maximum discharge current (within 15 s) & $48 \mathrm{~A}$ \\
Cut-off voltage range & $2.5-3.6 \mathrm{~V}$ \\
\hline
\end{tabular}

\subsection{Verification of the Reliability Criterion}

As verified before, the reliability criterion can get rid of unreliable parameters and keep the acquired parameters convergent under the constant current and pulse current conditions. Since the SOC estimation accuracy of the AEKF algorithm strongly depends on the model accuracy, we employ the AEKF algorithm to examine the reliability criterion. The UDDS cycle is also applied, and the results are presented in Figure 9. For convenience, the AEKF using the reliability criterion is expressed as AEKF $(R)$, while the AEKF (NO-R) stands for the algorithm without applying the criterion. As illustrated in Figure 9, the proposed criterion effectively improves the SOC estimation accuracy, and the time of SOC error convergence to less than $3 \%$ reduces to $500 \mathrm{~s}$. As the reliability criterion improves the accuracy of the model parameters at the beginning part, the AEKF algorithm can rapidly correct the initial SOC error and keep close to the real value. For a $\mathrm{LiFePO}_{4}$ battery, the optimal $\Delta$ equals 12 which is determined through experimental analysis.
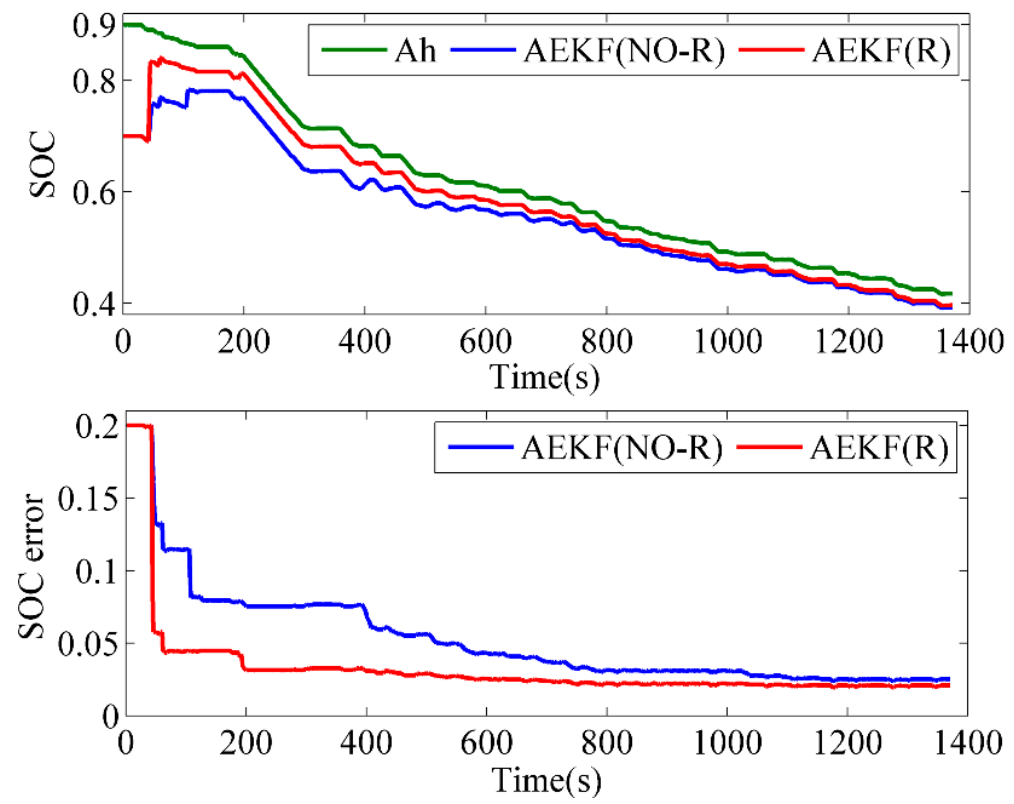

Figure 9. The SOC estimation results for evaluating the parameter reliability criterion. 


\subsection{Verification of the FAEKF Algorithm}

In the laboratory environment, the SOC profiles obtained by the Ampere-Hour integral method can be regarded as real SOC values for reference. The estimation results of AEKF using the proposed online identification strategy abbreviated as AEKF (online) are also presented to validate the improvement of the proposed FAEKF.

A sequence of experiments including pulse test, constant current test and driving cycle test are conducted to verify the algorithms. The pulse test profiles are shown in Figure 10a, and the SOC estimation results are presented in Figure 10b. Both algorithms have satisfactory estimation accuracy with most of the SOC estimation errors being less than 0.02. The mean error (ME) of AEKF (online) is 0.022 , while the ME of FAEKF is only 0.012. The FAEKF can gradually converge to the real SOC, while the AEKF (online) deviates from the real SOC all the time. The lower accuracy of the AEKF (online) indicates that the existing model errors are adverse to the SOC estimation. The FAEKF shows better performance than the AEKF (online) since it makes use of the prior knowledge to compensate the intrinsic model error. The constant current test inspects the algorithm's performance under continuous medium current output/input operation, such as running on the highway or executing a fast charging strategy. The test results are presented in Figure 11. For the FAEKF, the SOC estimation error at $1 \mathrm{C}$ current is less than 0.02 most of the time, and its ME is only 0.028 compared with 0.057 for the AEKF (Online). It is noted that the estimation error of the AEKF (Online) under $2 \mathrm{C}$ constant current discharge is evidently larger than that under $1 \mathrm{C}$ constant current discharge, which shows that a high battery current leads to a larger model error. Since the FAEKF considers the effect of the battery current directly, it shows better performance under both $1 \mathrm{C}$ and $2 \mathrm{C}$ constant current conditions.
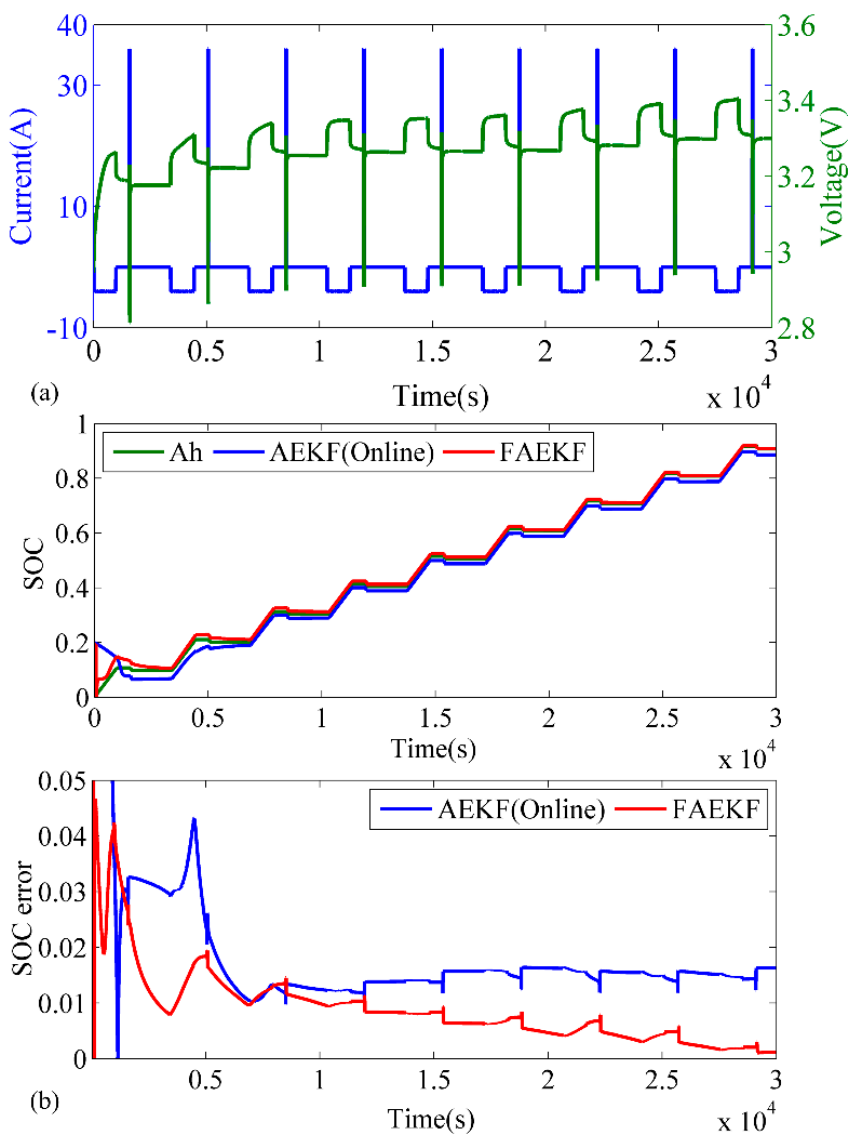

Figure 10. The SOC estimation results for pulse test: (a) current and voltage profiles; (b) SOC estimation results. 

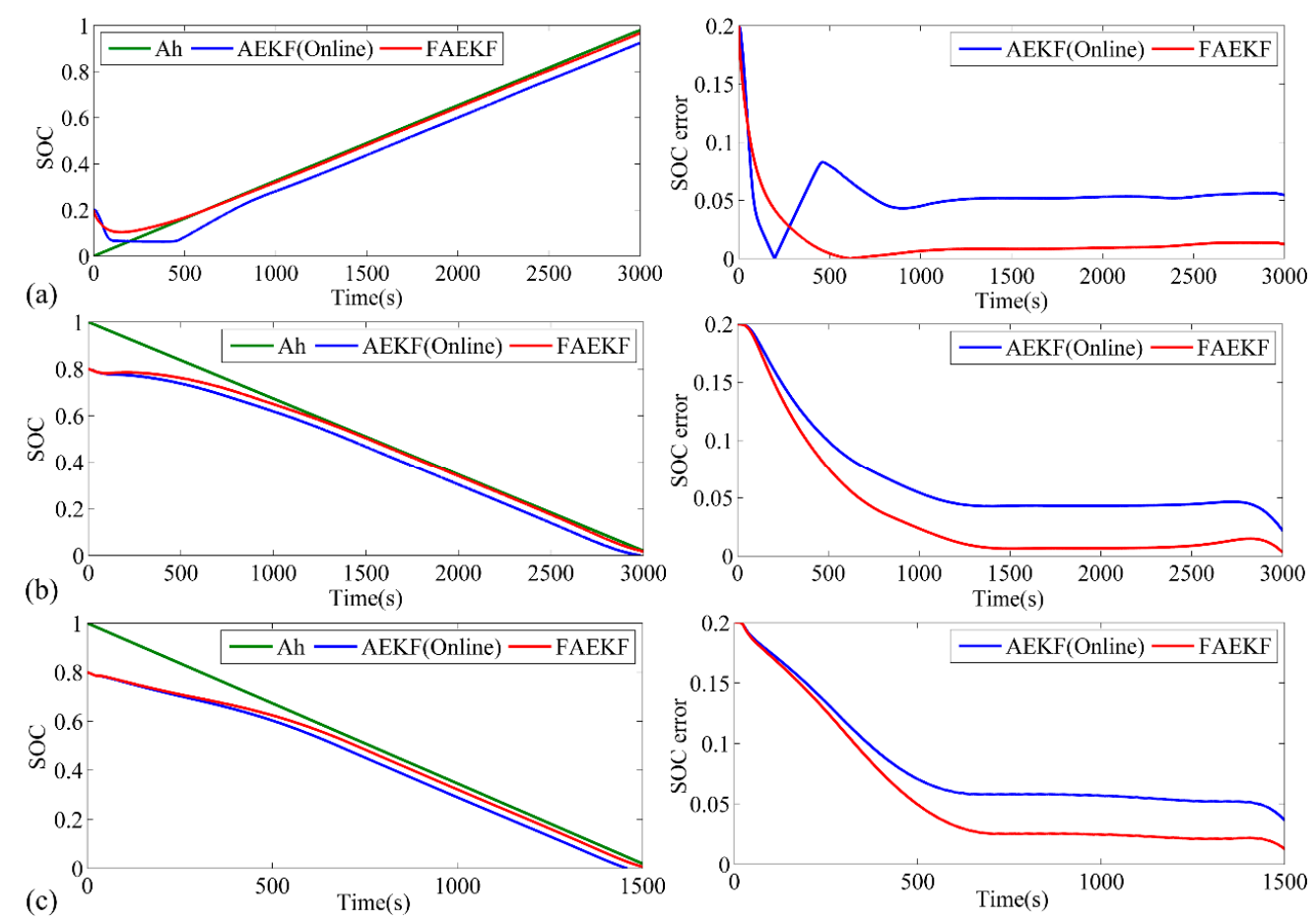

Figure 11. The SOC estimation results for constant current test. (a) $1 \mathrm{C}$ charge current; (b) $1 \mathrm{C}$ discharge current; (c) 2C discharge current.

The driving cycle test is necessary to validate that the proposed algorithm is effective when applied to vehicles. For validating the algorithms' correction ability for erroneous SOC, the initial SOC error is set as 0.2, and the corresponding results are presented in Figure 12a,b. Compared with the results of the constant current test, the SOC estimation accuracy is much better during the driving cycle. The results verify that the online identification method can obtain more precise parameters under the driving cycle conditions, since the frequent changing current can represent the complete dynamics of the battery system. Just because the accuracy of the model parameters has been improved, the AEKF (Online) and FAEKF can correct the initial SOC error and maintain the convergence in a timely fashion. The ME of the FAEKF are 0.019 for the initial $\mathrm{SOC}=0.9$ and 0.018 for the initial $\mathrm{SOC}=0.7$, while the ME of AEKF (Online) are 0.033 and 0.029 , respectively. It is remarkable that the FAEKF can converge to the real SOC after a period of time but the AEKF (Online) maintains a certain error all the time. The results confirm that the FAEKF can compensate the intrinsic model error and then further improve the SOC estimation accuracy.
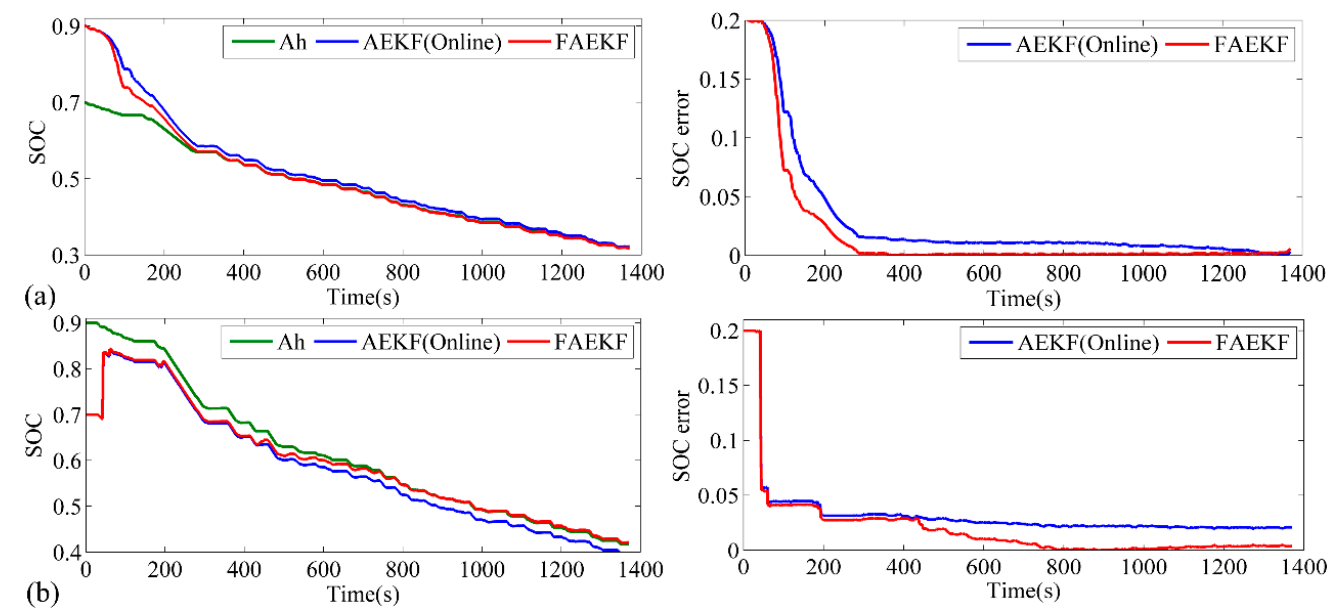

Figure 12. The SOC estimation results for UDDS cycle test: (a) initial SOC is 0.7 ; (b) initial SOC is 0.9 . 


\section{Conclusions}

With the consideration of reducing the computational complexity and conveniently integrating the online identification and SOC estimation algorithms into a BMS, the widely used Thevenin model is employed to model the battery dynamic behaviors in this paper. An improved ARMAX of the Thevenin model is derived, and the RELS method is employed to identify online the model parameters. Less model errors are acquired by the RELS method when compared with the commonly used RLS method. It is noted that the accuracy of online identification is susceptible to the complicated driving cycle. To evaluate the reliability of online parameters, a criterion based on the OCV error is proposed to extracts the believable parameters for states estimation.

Since the simplified ECM contains an intrinsic model error which is unfavorable to the SOC estimation, this paper proposes a fuzzy model to compensate the model error. Based on the verified prior knowledge, the fuzzy model is established to update the measurement noise covariance of Kalman filter in real time. The experiment results for $\mathrm{LiFePO}_{4}$ batteries show that the AEKF algorithm based on the proposed online identification strategy can obtain satisfactory SOC estimation accuracy under the driving cycle conditions, but relatively poor SOC estimation accuracy under pulse and constant current operations, while the proposed FAEKF presents better SOC estimation performance under the pulse current, constant current and the driving cycle operations. Though the methods proposed in this paper focus on the $\mathrm{LiFePO}_{4}$ battery, it can also apply to other types of battery after appropriate modifications, including recalibrating the parameter $\Delta$ and modifying the fuzzy rules.

Acknowledgments: This work is supported by National Key Technology R\&D Program under Grant 2013BAG03B01. The authors would like to express their appreciations for the fund.

Author Contributions: This research article has four authors. Zhongwei Deng and Yishan Cai conceived and designed the experiments; Hao Deng performed the experiments; Yishan Cai analyzed the data; Lin Yang contributed reagents/materials/analysis tools; Zhongwei Deng wrote the paper.

Conflicts of Interest: The authors declare no conflict of interest.

\section{References}

1. Waag, W.; Fleischer, C.; Sauer, D.U. Critical review of the methods for monitoring of lithium-ion batteries in electric and hybrid vehicles. J. Power Sources 2014, 258, 321-339. [CrossRef]

2. Lu, L.; Han, X.; Li, J.; Hua, J.; Ouyang, M. A review on the key issues for lithium-ion battery management in electric vehicles. J. Power Sources 2013, 226, 272-288. [CrossRef]

3. He, W.; Williard, N.; Osterman, M.; Pecht, M. Prognostics of lithium-ion batteries based on Dempster-Shafer theory and the Bayesian Monte Carlo method. J. Power Sources 2011, 196, 10314-10321. [CrossRef]

4. Wang, D.; Miao, Q.; Pecht, M. Prognostics of lithium-ion batteries based on relevance vectors and a conditional three-parameter capacity degradation model. J. Power Sources 2013, 239, 253-264. [CrossRef]

5. Guo, X.; Kang, L.; Yao, Y.; Huang, Z.; Li, W. Joint Estimation of the Electric Vehicle Power Battery State of Charge Based on the Least Squares Method and the Kalman Filter Algorithm. Energies 2016, 9, 100. [CrossRef]

6. Feng, F.; Lu, R.; Wei, G.; Zhu, C. Online estimation of model parameters and state of charge of LiFePO4 batteries using a novel open-circuit voltage at various ambient temperatures. Energies 2015, 8, 2950-2976. [CrossRef]

7. Xing, Y.; He, W.; Pecht, M.; Tsui, K.L. State of charge estimation of lithium-ion batteries using the open-circuit voltage at various ambient temperatures. Appl. Energy 2014, 113, 106-115. [CrossRef]

8. Charkhgard, M.; Farrokhi, M. State-of-charge estimation for lithium-ion batteries using neural networks and EKF. IEEE Trans. Ind. Electron. 2010, 57, 4178-4187. [CrossRef]

9. Wang, J.; Chen, Q.; Cao, B. Support vector machine based battery model for electric vehicles. Energy Convers. Manage. 2006, 47, 858-864.

10. Plett, G.L. Extended Kalman filtering for battery management systems of LiPB-based HEV battery packs: Part 2. Modeling and identification. J. Power Sources 2004, 134, 262-276. [CrossRef] 
11. He, H.; Xiong, R.; Zhang, X.; Sun, F.; Fan, J. State-of-charge estimation of the lithium-ion battery using an adaptive extended Kalman filter based on an improved Thevenin model. IEEE Trans. Veh. Technol. 2011, 60, 1461-1469.

12. He, W.; Williard, N.; Chen, C.; Pecht, M. State of charge estimation for electric vehicle batteries using unscented Kalman filtering. Microelectron. Reliab. 2013, 53, 840-847. [CrossRef]

13. Yang, F.; Xing, Y.; Wang, D.; Tsui, K.L. A comparative study of three model-based algorithms for estimating state-of-charge of lithium-ion batteries under a new combined dynamic loading profile. Appl. Energies 2016, 164, 387-399. [CrossRef]

14. Sun, F.; Hu, X.; Zou, Y.; Li, S. Adaptive unscented Kalman filtering for state of charge estimation of a lithium-ion battery for electric vehicles. Fuel Energy Abstr. 2011, 36, 3531-3540. [CrossRef]

15. Schwunk, S.; Armbruster, N.; Straub, S.; Kehl, J.; Vetter, M. Particle filter for state of charge and state of health estimation for lithium-iron phosphate batteries. J. Power Sources 2013, 239, 705-710. [CrossRef]

16. Lee, J.; Nam, O.; Cho, B.H. Li-ion battery SOC estimation method based on the reduced order extended Kalman filtering. J. Power Sources 2007, 174, 9-15. [CrossRef]

17. Mohamed, A.H.; Schwarz, K.P. Adaptive Kalman filtering for INS/GPS. J. Geod. 1999, 73, 193-203. [CrossRef]

18. Singh, P.; Vinjamuri, R.; Wang, X.; Reisner, D. Fuzzy logic modeling of EIS measurements on lithium-ion batteries. Electrochimica Acta 2006, 51, 1673-1679. [CrossRef]

19. Singh, P.; Fennie, C.; Reisner, D. Fuzzy logic modelling of state-of-charge and available capacity of nickel/metal hydride batteries. J. Power Sources 2004, 136, 322-333. [CrossRef]

20. Burgos, C.; Sáez, D.; Orchard, M.E.; Cárdenas, R. Fuzzy modelling for the state-of-charge estimation of lead-acid batteries. J. Power Sources 2015, 274, 355-366. [CrossRef]

21. Loebis, D.; Sutton, R.; Chudley, J.; Naeem, W. Adaptive tuning of a Kalman filter via fuzzy logic for an intelligent AUV navigation system. Control Eng. Pract. 2004, 12, 1531-1539. [CrossRef]

22. He, H.; Xiong, R.; Fan, J. Evaluation of lithium-ion battery equivalent circuit models for state of charge estimation by an experimental approach. Energies 2011, 4, 582-598. [CrossRef]

23. Hu, X.; Li, S.; Peng, H. A comparative study of equivalent circuit models for Li-ion batteries. J. Power Sources 2012, 198, 359-367. [CrossRef]

24. Xiong, R.; Sun, F.; Gong, X.; Gao, C. A data-driven based adaptive state of charge estimator of lithium-ion polymer battery used in electric vehicles. Appl. Energy 2014, 113, 1421-1433. [CrossRef]

25. Feng, T.; Yang, L.; Zhao, X.; Zhang, H.; Qiang, J. Online identification of lithium-ion battery parameters based on an improved equivalent-circuit model and its implementation on battery state-of-power prediction. J. Power Sources 2015, 281, 192-203. [CrossRef]

26. Plett, G.L. Extended Kalman filtering for battery management systems of LiPB-based HEV battery packs: Part 3. State and parameter estimation. J. Power Sources 2004, 134, 277-292. [CrossRef]

27. Bergveld, H.J.; Kruijt, W.S.; Notten, P.H. Battery Management Systems: Design by Modelling; Philips Research Book Series; Kluwer Academic Publishers: Boston, MA, USA, 2002.

28. Snihir, I.; Rey, W.; Verbitskiy, E.; Belfadhel-Ayeb, A.; Notten, P.H. Battery open-circuit voltage estimation by a method of statistical analysis. J. Power Sources 2006, 159, 1484-1487. [CrossRef]

29. Coleman, M.; Hurley, W.G.; Lee, C.K. An improved battery characterization method using a two-pulse load test. IEEE Trans. Energy Convers. 2008, 23, 708-713. [CrossRef]

30. Han, J.; Kim, D.; Sunwoo, M. State-of-charge estimation of lead-acid batteries using an adaptive extended Kalman filter. J. Power Sources 2009, 188, 606-612. [CrossRef]

(C) 2016 by the authors; licensee MDPI, Basel, Switzerland. This article is an open access article distributed under the terms and conditions of the Creative Commons Attribution (CC-BY) license (http://creativecommons.org/licenses/by/4.0/). 